ARTÍCULOS ORIGINALES Rev Chil Salud Pública 2014; Vol 18 (2): 161-172
Julia Becaria Coquet* Sabrina Mariné Juárez Mariel Alejandra Flores Sonia Alejandra Pou Laura Rosana Aballay María del Pilar Díaz Escuela de Nutrición. Facultad de Ciencias Médicas. Universidad Nacional de Córdoba. *juliabc@fcm.unc.edu.ar

\section{IDENTIFICACIÓN DE FACTORES DE CONFUSIÓN EN EL ESTUDIO DE LA RELACIÓN CÁNCER COLORRECTAL-DIETA}

\author{
IDENTIFYING CONFOUNDING FACTORS IN STUDIES OF THE \\ RELATIONSHIP BETWEEN COLORECTAL CANCER AND DIET
}

\section{RESUMEN}

Introducción. La dieta se reconoce como segunda causa evitable relacionada con el desarrollo de cáncer. No obstante, dado su naturaleza multicausal, al estudiar la relación cáncer-dieta deben considerarse otros factores con potencial efecto confusor para evitar sesgos en estimaciones de riesgo.

Objetivos. a) Identificar el efecto confundente del nivel de actividad fisica, hábito de fumar y nivel socioeconómico en la relación cáncer colorrectal (CCR) y dieta; b) Valorar el riesgo de factores alimentarios asociados a $C C R$, considerando las variables confundentes identificadas.

Metodología. Se condujo un estudio caso-control ( $n=319 ; 102$ casos de CCR, 217 controles) en Córdoba, Argentina (2006-2011). Se realizó un análisis bivariado entre variables alimentarias de interés y presencia de CCR, estimando ORs como medida de asociación. Luego, mediante análisis de Mantel-Haenszel, se estratificó por potenciales variables confundentes. Finalmente, se construyeron modelos de regresión logística múltiple, incluyendo las confundentes.

Resultados. Se verificó efecto confusor del nivel socioeconómico en relación al consumo de carnes rojas cocidas, fibra y etanol, y de la actividad física en cuanto al consumo de fibra alimentaria. Controlando por dichos efectos, no se encontró asociación (OR 0,71; IC95\% 0,31-1,62) entre la ingesta de fibra y la patología, y se observó un efecto promotor (OR 1,75; IC95\% 0,95$2,60)$ del nivel socioeconómico bajo y de la ingesta energética diaria (OR 1,0003; IC95\% 1,00008-1,0006).

Conclusión. Se reconoce el nivel socioeconómico y la actividad física como potenciales variables confusoras en el estudio de la relación CCR y alimentación en Argentina. Se recomienda considerarlas como variables de ajuste al realizar análisis de riesgos alimentarios.

Palabras clave: cáncer colorrectal, factores de confusión, fibra alimentaria, carnes rojas, Argentina.

\footnotetext{
* Financiado por Agencia Nacional de Promoción Científica y Tecnología. Fondo para la Investigación Científica y Tecnológica (FONCyT PICT 2008 1814). Ministerio de Ciencia, Tecnología e Innovación Productiva, Argentina.
} 


\section{ABSTRACT}

Introduction. Diet is the second preventable cause related to the development of cancer. Given its multi-causal nature, in studying the relationship between cancer and diet, other factors with potential confounding effect must be considered to avoid bias in risk estimates. Objectives: a) Identifying the confounding effect of physical activity level, smoking babits and socioeconomic status in the relationship between colorectal cancer and cooked red meat, fiber and alcohol intake; b) Assessing the effect of dietary factors on the occurrence of colorectal cancer, considering the confounding variables identified.

Methods. A case-control study was conducted (102 cases with colorectal cancer and 217 controls) in Cordoba, Argentina, over 2006-2011. A bivariate analysis, between food variables and the presence of colorectal cancer, and a Mantel-Heanzel analysis, stratifying by the potential confounders, were conducted. Finally, multiple logistic regression models were constructed, including the confounding variables.

Results. Confounding effect of the socioeconomic status related to cooked red meat, fiber and alcohol intake, and physical activity level was verified. There was no association between fiber intake and colorectal cancer $(O R$ 0,71; IC95\% 0,31-1,62), while a promoting effect of low socioeconomic status (OR 1,75; IC95\% 0,95-2,60), and daily energy intake (OR 1,0003; IC95\% 1,00008-1,0006) were found.

Conclusion. It is recommended to consider socioeconomic status and physical activity as adjusted factors when conducting food risk analysis in the study of the relationship between colorectal cancer and diet in Argentina.

Key words: colorectal cancer, confounding factors, dietary fiber, red meat, Argentina.

\section{INTRODUCCIÓN}

El cáncer se ha convertido en un problema de salud pública en todo el mundo, que afecta a personas de todas las edades y condiciones socioeconómicas. Los factores que dan lugar a su desarrollo pueden ser tanto endógenos como exógenos. Entre el $80-90 \%$ de los tumores malignos en adultos son causados por factores ambientales, y se estima que el 35\% de ellos están relacionados con la alimentación. Esto significa que la mayoría de los cánceres son, por lo menos en teoría, prevenibles. ${ }^{1,2}$ El cáncer es la segunda causa de muerte en países desarrollados y figura entre las tres principales en países en desarrollo. ${ }^{2}$ En Argentina, es la segunda causa de muerte por enfermedad y poco se conoce sobre su incidencia. Díaz et al. (2010), ${ }^{4}$ reportan tasas de incidencia totales estandarizadas y ajustadas por edad, iguales a $263.53 \pm 138.34 \mathrm{y}$ $200.45 \pm 98.30$ (x100.000 hab/año) para hombres y mujeres, respectivamente.
El cáncer colorrectal (CCR) se presenta habitualmente entre la sexta y octava década de vida, e incluso antes de los 50 años en las formas hereditarias. Más del $95 \%$ de los CCR corresponden a adenocarcinomas. ${ }^{5}$ Es el tercer tipo más frecuente a nivel mundial, representando cerca del $9 \%$ del total de cánceres. En Argentina, la tasa de incidencia de CCR estandarizada por edad (TIE) es igual a $20,4^{6}$ y en la provincia de Córdoba, 19,4 y 12,5 en hombres y mujeres respectivamente.

Existe evidencia acerca de la asociación directa entre la ocurrencia de CCR y la ingesta de carnes rojas, procesadas y alcohol. ${ }^{8-11}$ Respecto a las primeras, su consumo se asocia a un aumento de carcinógenos en la dieta, como las aminas aromáticas heterocíclicas e hidrocarburos aromáticos policíclicos derivados de reacciones térmicas durante su cocción a altas temperaturas. ${ }^{8}$ Las carnes procesadas (fiambres, embutidos) contienen niveles altos de nitritos y nitratos que contribuyen a la formación de nitrosaminas capaces de da- 
ñar el ADN. ${ }^{8-10}$ Se ha demostrado que el nitrito y el nitrato, las aminas heterocíclicas y el total de mutágenos procedentes de la carne contribuyen de forma modesta, pero consistentemente, con el riesgo de padecer pólipos adenomatosos. ${ }^{9}$ En cuanto al etanol, diversos estudios afirman que puede facilitar el ingreso de otras moléculas cancerígenas en las células de la mucosa, debido a que actúa como un solvente. Su presencia, además, dificulta la eficiencia en la reparación de las mutaciones específicas del ADN, como las inducidas por el tabaco. Asimismo, algunos metabolitos de este, como el acetaldehído, pueden ser cancerígenos. ${ }^{11}$

Por otra parte, la ingesta de alimentos ricos en fibra se reportan asociados de manera inversa con el riesgo de CCR. ${ }^{9}, 12$ En general, se sugieren como mecanismos subyacentes ligados a su efecto protector el hecho de que la fibra disminuye el tiempo de tránsito intestinal, aumenta el volumen de las heces $\mathrm{y}$, por fermentación, interviene en la liberación de ácidos grasos de cadena corta, como el butirato. Este último, induce apoptosis, detención del ciclo celular y diferenciación celular. $^{1,9}$

La epidemiología nutricional es una disciplina científica cuyo objetivo primario es valorar la dieta de las poblaciones como un factor de exposición asociado a la mayor o menor ocurrencia de enfermedad. ${ }^{13}$ Esto se reconoce como un verdadero desafío analítico, dada la complejidad de la dieta humana, ${ }^{14}$ sumado a la multicausalidad de muchas enfermedades, y en especial las crónicas. De hecho, además de las variables consideradas en el estudio de la relación entre un factor de exposición como la dieta, y la presencia de una enfermedad (p.e. cáncer), existen otros intervinientes que inducen, potencian, debilitan o eliminan la asociación observada. Estos factores han sido denominados como de confusión o confundentes. ${ }^{15}$ El fenómeno de confusión puede generar sesgos en las estimaciones de riesgo, ya que sin su control o ajuste analítico la interpretación de los resultados obtenidos será errónea. ${ }^{16,17}$ Este se presenta con más frecuencia en estudios observacionales (como los estudios caso-control) que en experimentales. La identificación de variables confundentes es fundamental para mejorar las estimaciones estadísticas en el estudio de la relación cáncer-dieta, a los fines de poder establecer relaciones causales certeras que permitan tomar decisiones acertadas en el campo de la Salud Pública.

Existe evidencia que respalda la asociación de las variables confundentes con las variables de exposición alimentaria propuestas y la enfermedad estudiada. ${ }^{18-23}$ No obstante, no se encontraron antecedentes de estudios similares al presente, que verifiquen previamente la significación estadística del efecto de confusión de las variables seleccionadas. Se destaca además que los hábitos alimentarios de la población argentina en general, y de Córdoba en particular, presentan ciertas características propias de esta región, como el elevado consumo de carnes y bajo consumo de fibras, ${ }^{24,25}$ que han sido incluso asociadas al riesgo de cáncer en estudios anteriores, ${ }^{24,26-29}$ lo cual constituye un escenario atractivo para estudios epidemiológicos en esta temática.

El presente estudio propuso, en primera instancia, identificar variables confundentes en el estudio de la relación entre CCR y factores alimentarios específicos, como la ingesta de fibra, carnes rojas cocidas, y alcohol, y a partir de ello, valorar el efecto de dichos factores alimentarios en la ocurrencia de esta patología, considerando la influencia de las variables confundentes identificadas, en adultos de la provincia de Córdoba (Argentina).

Para ello se plantearon las siguientes hipótesis: a) existe un efecto de confusión del nivel de actividad física y el nivel socioeconómico en el estudio de las relaciones consumo de fibra y CCR y entre el consumo de carnes rojas cocidas y CCR en la población estudiada, y b) el nivel de actividad física, nivel socioeconómico y el hábito de fumar intervienen como variables de confusión en el estudio de la relación consumo de alcohol y CCR.

\section{MATERIAL Y MÉTODOS}

Se realizó un estudio de tipo caso-control en la Provincia de Córdoba (Argentina), durante el 
período 2006-2011. Se conformó una muestra de 319 sujetos: 102 casos incidentes de CCR y 217 controles. Los casos (incidentes) fueron individuos que recibieron diagnóstico histopatológicamente confirmado de adenocarcinoma de colon o recto (CIE C18-C20) en hospitales e instituciones de salud de la Provincia de Córdoba (Argentina) durante el período de estudio, y los controles (dos a tres por cada caso) sujetos sin la enfermedad, seleccionados aleatoriamente en las localidades de procedencia de cada caso, siendo luego apareados de acuerdo a las características biológicas (sexo y edad: \pm 5 años) de estos.

El tamaño de la muestra se estimó considerando la tasa de incidencia de cáncer colorrectal en Córdoba (casos esperados por año); además se tuvieron en cuenta las necesidades de los tests estadísticos para trabajar con una potencia de por lo menos $95 \%$ : mínimo dos controles por cada caso. ${ }^{30}$

Los criterios de exclusión definidos fueron la modificación en los hábitos alimentarios por la presencia de enfermedades crónicas (como cardiopatías, diabetes), por costumbre, religión o prescripción médica, y la presencia de antecedentes personales de patologías oncológicas en los controles.

Los sujetos con diagnóstico primario de CCR fueron seleccionados a partir de registros de atención de pacientes de los servicios de oncología y gastroenterología, actas de internación e historias clínicas, de los principales hospitales públicos y privados de la ciudad de Córdoba (capital) y localidades cabecera del interior provincial. La recolección de la información se realizó mediante entrevistas personales y domiciliarias, a los sujetos casos identificados y sus respectivos controles, realizadas por profesionales capacitados especialmente para dicha tarea. El instrumento consistió en un cuestionario estructurado, validado por Navarro y cols. para estudios epidemiológicos sobre cáncer en Argentina, ${ }^{31}$ el cual recaba información acerca de datos personales, antropométricos, de estilos de vida (hábito de fumar, consumo de alcohol, actividad física) y referidos a la alimentación habitual. Específicamente, para la valoración de la ingesta alimentaria incluye un cuestionario de frecuencia alimentaria de 127 ítems alimentarios, sus formas de preparación, frecuencia de consumo y el tamaño de las porciones. Este cuestionario indaga sobre la alimentación referida a cinco años previos al momento del diagnóstico de los casos entrevistados, y cinco años previos al momento de la encuesta para los controles. Se utilizó como material complementario un atlas con fotografías estandarizadas de alimentos, también validado. ${ }^{32} \mathrm{El}$ cálculo de la ingesta alimentaria-nutricional fue realizado mediante el software de análisis nutricional Nutrio $2 .^{33}$

La clasificación de la actividad física se basó en el criterio del IPAQ,,34 colapsándose las categorías de actividad moderada e intensa en la que denominamos activo; para la definición del nivel socioeconómico se consideró la cantidad de personas que aportan en el hogar, posesión de bienes y servicios (computadora, conexión a internet, tarjeta de débito, automóvil), tipo de atención médica (privada o pública) y nivel educativo del principal sostén del hogar. El estado nutricional se definió basados en los datos antropométricos (peso y talla) autorreportados por los sujetos estudiados. La clasificación de la variable se basó en el criterio de la OMS. ${ }^{35}$

Cada individuo proporcionó su conformidad para participar en la investigación mediante un consentimiento informado. Este trabajo se condujo respetando las normas éticas incluidas en la Declaración de Helsinki 1995 (y su versión revisada, Tokio 2004). ${ }^{36}$ Fue evaluado por el comité de ética correspondiente e inscrito en el Registro Provincial de Investigaciones en Salud (RePIS 044/10 y Enmienda 044/10).

El procesamiento de la información recabada fue realizado por los mismos encuestadores, debidamente entrenados para ello, con supervisión permanente por parte de un investigador responsable, encargado de efectuar la revisión de las encuestas almacenadas en la base de datos (verificación de inconsistencia, correspondencias en la codificación, etcétera). Por otra parte, para evitar errores de digitación en el ingreso de datos, el cuestionario de frecuencia alimentaria presenta líneas de 
totales, por cada página, de la cantidad de alimentos con datos sobre frecuencia de consumo. Estos totales son completados por el encuestador a mano. Luego el software de ingreso de datos alimentarios realiza el mismo cálculo, automáticamente, permitiendo verificar que los totales cuadren, evitando así la omisión o duplicación de datos en el procedimiento de cargado de información.

\section{ANÁLISIS ESTADÍSTICOS}

Fue llevado a cabo un análisis bivariado (crudo) entre variables alimentarias seleccionadas y la presencia de CCR, y estimados los Odd Ratios (OR), como medida de asociación. La variable respuesta (outcome) fue la presencia de CCR, definiendo como covariables alimentarias los consumos de fibra (bajo consumo: $\leq 15 \mathrm{~g} /$ día; consumo medio:15,1-25 g/ día; alto consumo: $>25 \mathrm{~g} /$ día), de carnes rojas cocidas ( $\leq 250 \mathrm{~g} /$ día o $>250 \mathrm{~g} /$ día), y de alcohol ( $\leq 20 \mathrm{~g}$ etanol/día, $>20 \mathrm{~g}$ etanol/día). El test de Mantel-Haenszel, estratificando por las potenciales variables confundentes: actividad física (activo y sedentario) y nivel socioeconómico (medio-alto y bajo) fue usado a partir de lo anterior. En el caso de consumo de etanol, se estudió adicionalmente, el efecto confusor de la variable hábito de fumar (fumadores y no fumadores).

Para la estimación de los riesgos asociados a las covariables, se construyeron modelos de regresión logística múltiple, ${ }^{37}$ estableciendo como variable outcome la presencia-ausencia de enfermedad (CCR). Como variables de ajuste se incluyeron las variables alimentarias de interés (consumo de fibra, carnes rojas cocidas y etanol), las variables identificadas como confundentes en este trabajo (nivel socioeconómico y nivel de actividad física), y otras con reconocida asociación con CCR, como el estado nutricional (sobrepeso-no sobrepeso según Índice de Masa Corporal, $\left.\geq 25 \mathrm{~kg} / \mathrm{m}^{2}\right)^{35}$ y el valor energético total (terciles de consumo de Cal/día). Se establece como criterio para considerar que existe un efecto estadísticamente significativo, un nivel $\alpha$ igual o mayor a 0,05 .
Todos los análisis estadísticos se realizaron en el software estadístico STATA, version 11 (Statacorp LP. Stata 11.0. College Station, TX: USA, 1990).

\section{RESULTADOS}

De un total de 319 personas encuestadas, un $46,1 \%$ fueron mujeres, presentando una media de edad de 62,23 años (DE, desviación estándar: 14,59 años). La Tabla 1 describe las características biosocioculturales y de exposición de casos y controles. En relación al nivel socioeconómico, se encontró asociación significativa $(\mathrm{p}=0,02)$ con la presencia de la enfermedad; el $60,78 \%$ de los individuos con la enfermedad pertenecía al nivel socioeconómico bajo, mientras que, entre los controles, este porcentaje fue del 46,08\%. Respecto a la presencia de antecedentes familiares de CCR, el $19,61 \%$ de individuos del grupo casos y solo un $4,15 \%$ de los sujetos controles manifestaron haber tenido algún familiar con la patología, siendo esta diferencia estadísticamente significativa $(\mathrm{p}<0,01)$.

El consumo de fibra no estuvo asociado con el CCR (consumo medio, $p=0,07$, $\mathrm{OR}=1,70$, y consumo alto de fibra, $\mathrm{p}=0,27$, $\mathrm{OR}=1,43$; respecto del consumo bajo). $\mathrm{Al}$ realizar el análisis estratificado en relación al nivel socioeconómico, se modificaron los valores de OR respecto al análisis crudo (Tabla 2). Esto indicaría que existe un efecto de confusión del NSE en la relación entre ingesta de fibra y la presencia de CCR. El nivel de actividad física también mostró un efecto confundente en la relación consumo de fibra y CCR (Tabla 2).

Respecto a la ingesta de carnes rojas cocidas, en relación a la enfermedad, se pudo deducir un efecto de confusión del NSE (Tabla 3). En el análisis crudo se observa que un consumo mayor a $250 \mathrm{~g} /$ día de carnes rojas cocidas presenta un $\mathrm{OR}=1,51(\mathrm{p}=0,10)$, respecto al grupo de menor consumo. Al realizar el análisis estratificado en relación al nivel socioeconómico, se modificaron los OR respecto al análisis crudo (nivel socioeconómico medio alto $\mathrm{p}=0,85, \mathrm{OR}=0.92$; nivel bajo 
Tabla 1. Distribución de frecuencias de las variables biosocioculturales y alimentarias de exposición, según casos y controles. Provincia de Córdoba. Año 2010-2011

\begin{tabular}{|c|c|c|c|}
\hline & Controles & Casos & Totales \\
\hline \multirow{2}{*}{ Características } & $\mathrm{n}=\mathbf{2 1 7}$ & $\mathrm{n}=102$ & $\mathrm{n}=319$ \\
\hline & $\mathrm{n}(\%)$ & $\mathrm{n}(\%)$ & $\mathrm{n}(\%)$ \\
\hline \multicolumn{4}{|l|}{ Nivel socioeconómico } \\
\hline Bajo* & $100(46,06)$ & $62(60,78)$ & $162(50,78)$ \\
\hline Medio-Alto & $117(53,92)$ & $40(39,22)$ & $157(49,12)$ \\
\hline \multicolumn{4}{|l|}{ Estado nutricional } \\
\hline Bajo peso & $9(4,14)$ & $5(4,90)$ & $14(4,40)$ \\
\hline Normal & $80(36,86)$ & $36(35,30)$ & $116(36,40)$ \\
\hline Preobesidad & $84(38,70)$ & $35(34,30)$ & $119(37,30)$ \\
\hline Obesidad & $44(20,30)$ & $26(25,50)$ & $70(21,90)$ \\
\hline \multicolumn{4}{|l|}{ Nivel de actividad física } \\
\hline Activo & $93(42,86)$ & $43(42,16)$ & $136(42,63)$ \\
\hline Sedentario & $124(57,14)$ & $59(57,84)$ & $183(57,37)$ \\
\hline \multicolumn{4}{|l|}{ Hábito de fumar } \\
\hline No fumador & $87(40,10)$ & $48(47,05)$ & $135(42,32)$ \\
\hline Fumador & $130(59,9)$ & $54(52,95)$ & $184(57,68)$ \\
\hline \multicolumn{4}{|c|}{ Antecedentes familiares de cáncer colorrectal } \\
\hline Con antecedentes* & $9(4,15)$ & $20(19,61)$ & $29(9,09)$ \\
\hline Sin antecedentes & $208(95,85)$ & $82(80,39)$ & $290(90,91)$ \\
\hline \multicolumn{4}{|l|}{ Consumo de fibra } \\
\hline Bajo & $74(34,10)$ & $25(24,51)$ & $99(31,03)$ \\
\hline Medio & $85(39,17)$ & $49(48,04)$ & $134(42,01)$ \\
\hline Alto & $58(26,73)$ & $28(27,45)$ & $86(26,96)$ \\
\hline \multicolumn{4}{|l|}{ Consumo de etanol (g/día) } \\
\hline$\leq 20$ & $143(65,90)$ & $63(61,76)$ & $206(64,58)$ \\
\hline$>20$ & $74(34,10)$ & $39(38,24)$ & $113(35,42)$ \\
\hline \multicolumn{4}{|c|}{ Consumo de carnes rojas (g/día) } \\
\hline$\leq 250$ & $152(70,05)$ & $62(60,78)$ & $214(67,08)$ \\
\hline$>250$ & $65(29,95)$ & $40(39,22)$ & $105(32,92)$ \\
\hline \multicolumn{4}{|l|}{ Valor energético total } \\
\hline & Media (DE) & Media (DE) & Media (DE) \\
\hline Cal/día & $3121(1311)$ & $3703(1557)$ & $3307(1418)$ \\
\hline
\end{tabular}

DE, desvío estándar, *p $<0,05$

$\mathrm{p}=0,04, \mathrm{OR}=0,95$; en relación a la referencia respectivamente).

$\mathrm{Al}$ analizar la variable de exposición consumo de etanol y su asociación con CCR (Tabla 4), se puede observar en el análisis crudo un $\mathrm{OR}=1,20$ para un consumo mayor a $20 \mathrm{~g} /$ día respecto al grupo de referencia. Solo se identificó efecto confundente de la variable nivel socioeconómico (nivel socioeconómico medio-alto $\mathrm{p}=0,08, \mathrm{OR}=1,92$; $\mathrm{y}$ nivel bajo $\mathrm{p}=0,66, \mathrm{OR}=0,86$; respecto a los grupos de referencia).

Controlando los mencionados efectos de confusión, no se encontró una asociación
(OR 0,73; IC 95\% 0,33-1,60) de la ingesta de fibra con la patología, y se observó un efecto promotor (OR 1,74; IC 95\% 1,04-2,90) del NSE bajo y de la ingesta energética diaria (Tercil Superior, OR 3,07; IC 95\% 1,27-7,43) sobre la ocurrencia de CCR (Tabla 5).

\section{DISCUSIÓN}

En este estudio se evidenció un efecto confusor de la variable nivel socioeconómico en relación a la ingesta de carnes rojas cocidas, fibra y etanol y el CCR, y de la actividad 
Tabla 2. Estimación de medidas de asociación (ORs) entre el consumo de fibra y el cáncer colorrectal. Análisis crudo y estratificado por variables de interés. Estudio caso-control. Provincia de Córdoba. Año 2010-2011

\begin{tabular}{|c|c|c|c|c|c|}
\hline \multicolumn{6}{|l|}{ Análisis crudo } \\
\hline \multicolumn{2}{|l|}{ Consumo de fibra (g/día) } & Controles (n) & Casos (n) & OR (IC) & Valor $\mathrm{p}$ \\
\hline \multicolumn{2}{|l|}{ Bajo } & 74 & 25 & Referencia & \\
\hline \multicolumn{2}{|l|}{ Medio } & 85 & 49 & $1,71(0,96-3,04)$ & 0,07 \\
\hline \multicolumn{2}{|l|}{ Alto } & 58 & 28 & $1,43(0,75-2,72)$ & 0,27 \\
\hline \multicolumn{2}{|l|}{ Totales } & 217 & 102 & & \\
\hline \multicolumn{6}{|c|}{ Análisis estratificado por nivel socioeconómico } \\
\hline Nivel Socioeconómico & Consumo de Fibra (g/día) & Controles (n) & Casos (n) & OR (IC) & Valor $\mathrm{p}$ \\
\hline \multirow[t]{3}{*}{ Medio-Alto } & Bajo & 39 & 12 & Referencia & \\
\hline & Medio & 48 & 20 & $1,35(0,59-3,12)$ & 0,47 \\
\hline & Alto & 30 & 8 & $0,87(0,31-2,40)$ & 0,78 \\
\hline \multirow[t]{3}{*}{ Bajo } & Bajo & 35 & 13 & Referencia & \\
\hline & Medio & 37 & 29 & $2,11(0,93-4,77)$ & 0,67 \\
\hline & Alto & 28 & 20 & $1,92(0,80-4,60)$ & 0,13 \\
\hline \multicolumn{6}{|c|}{ Análisis estratificado por actividad física } \\
\hline Actividad física & Consumo de Fibra (g/día) & Controles (n) & Casos (n) & OR (IC) & Valor $\mathrm{p}$ \\
\hline \multirow[t]{3}{*}{ Activo } & Bajo & 31 & 9 & Referencia & \\
\hline & Medio & 39 & 21 & $1,85(0,73-4,68)$ & 0,18 \\
\hline & Alto & 20 & 13 & $2,24(0,79-6,36)$ & 0,12 \\
\hline \multirow[t]{3}{*}{ Sedentario } & Bajo & 43 & 16 & Referencia & \\
\hline & Medio & 46 & 28 & $1,63(0,77-3,46)$ & 0,19 \\
\hline & Alto & 38 & 15 & $1,06(0,46-2,44)$ & 0,88 \\
\hline
\end{tabular}

Tabla 3. Estimación de medidas de asociación (ORs) entre el consumo de carnes rojas cocidas y el cáncer colorrectal. Análisis crudo y estratificado por variables de interés. Estudio caso-control. Provincia de Córdoba. Año 2010-2011

\begin{tabular}{|c|c|c|c|c|c|}
\hline \multicolumn{6}{|l|}{ Análisis crudo } \\
\hline \multicolumn{2}{|c|}{ Consumo de carnes rojas Cocidas (g/día) } & Controles (n) & Casos (n) & OR (IC) & Valor p \\
\hline \multicolumn{2}{|l|}{$\leq 250$} & 152 & 62 & Referencia & \\
\hline \multicolumn{2}{|l|}{$>250$} & 65 & 40 & $1.51(0,92-2,47)$ & 0.10 \\
\hline \multicolumn{2}{|l|}{ Totales } & 217 & 102 & & \\
\hline \multicolumn{6}{|c|}{ Análisis estratificado por nivel socioeconómico } \\
\hline Nivel socioeconómico & $\begin{array}{l}\text { Consumo de carnes } \\
\text { rojas cocidas (g/día) }\end{array}$ & Controles (n) & Casos (n) & OR (IC) & Valor $\mathrm{p}$ \\
\hline \multirow[t]{2}{*}{ Medio-Alto } & $\leq 250$ & 83 & 29 & Referencia & \\
\hline & $>250$ & 34 & 11 & $0,92(0,41-2,07)$ & 0,85 \\
\hline \multirow[t]{2}{*}{ Bajo } & $\leq 250$ & 69 & 33 & Referencia & \\
\hline & $>250$ & 31 & 29 & $0,95(1,01-3,80)$ & 0,04 \\
\hline \multicolumn{6}{|c|}{ Análisis estratificado por actividad física } \\
\hline Actividad física & $\begin{array}{l}\text { Consumo de carnes } \\
\text { rojas cocidas (g/día) }\end{array}$ & Controles (n) & Casos (n) & OR & Valor $\mathrm{p}$ \\
\hline \multirow[t]{2}{*}{ Activo } & $\leq 250$ & 71 & 29 & Referencia & \\
\hline & $>250$ & 19 & 14 & $1,80(0,79-4,11)$ & 0,15 \\
\hline \multirow[t]{2}{*}{ Sedentario } & $\leq 250$ & 81 & 33 & Referencia & \\
\hline & $>250$ & 46 & 26 & $1,39(074-2,61)$ & 0,31 \\
\hline
\end{tabular}


Tabla 4. Estimación de medidas de asociación (ORs) entre el consumo de etanol y el cáncer colorrectal. Análisis crudo y estratificado por variables de interés. Estudio caso-control. Provincia de Córdoba. Año 2010-2011

\begin{tabular}{|c|c|c|c|c|c|}
\hline \multicolumn{6}{|l|}{ Análisis crudo } \\
\hline \multicolumn{2}{|c|}{ Consumo de etanol (g/día) } & Controles (n) & $\operatorname{Casos}(\mathbf{n})$ & OR (IC) & Valor p \\
\hline \multicolumn{2}{|l|}{$\leq 20$} & 143 & 63 & Referencia & \\
\hline \multicolumn{2}{|l|}{$>20$} & 74 & 39 & $1,20(0,73-1,95)$ & 0,47 \\
\hline \multicolumn{2}{|l|}{ Totales } & 217 & 102 & & \\
\hline \multicolumn{6}{|c|}{ Análisis estratificado por nivel socioeconómico } \\
\hline Nivel socioeconómico & Consumo de etanol(g/día) & Controles (n) & Casos (n) & OR (IC) & Valor $\mathrm{p}$ \\
\hline \multirow[t]{2}{*}{ Medio-Alto } & $\leq 20$ & 77 & 20 & Referencia & \\
\hline & $>20$ & 40 & 20 & $1,92(0,92-4,02)$ & 0,08 \\
\hline \multirow[t]{2}{*}{ Bajo } & $\leq 20$ & 66 & 43 & Referencia & \\
\hline & $>20$ & 34 & 19 & $0,86(0,43-1,70)$ & 0,66 \\
\hline \multicolumn{6}{|c|}{ Análisis estratificado por actividad física } \\
\hline Actividad física & Consumo de etanol (g/día) & Controles (n) & Casos (n) & OR & Valor p \\
\hline \multirow[t]{2}{*}{ Activo } & $\leq 20$ & 64 & 30 & Referencia & \\
\hline & $>20$ & 26 & 13 & $1,07(0,48-2,36)$ & 0,87 \\
\hline \multirow[t]{2}{*}{ Sedentario } & $\leq 20$ & 79 & 33 & Referencia & \\
\hline & $>20$ & 48 & 26 & $1,30(0,69-2,43)$ & 0,42 \\
\hline \multicolumn{6}{|c|}{ Análisis estratificado por hábito de fumar } \\
\hline Hábito de fumar & Consumo de etanol (g/día) & Controles (n) & Casos (n) & OR (IC) & Valor p \\
\hline \multirow[t]{2}{*}{ No fumadores } & $\leq 20$ & 68 & 35 & Referencia & \\
\hline & $>20$ & 19 & 13 & $1,33(0,60-3,02)$ & 0,50 \\
\hline \multirow[t]{2}{*}{ Fumadores } & $\leq 20$ & 75 & 28 & Referencia & \\
\hline & $>20$ & 55 & 26 & $1,27(0,67-2,40)$ & 0,47 \\
\hline
\end{tabular}

Tabla 5. Resultados del modelo de regresión logística múltiple para la presencia-ausencia de CCR como outcome. Estudio caso-control. Provincia de Córdoba. Año 2010-2012.

\begin{tabular}{|c|c|c|c|c|}
\hline Variables de ajuste & & OR & IC $(95 \%)$ & Valor $\mathrm{p}$ \\
\hline \multirow{3}{*}{ Consumo de Fibra } & Bajo & Referencia & & \\
\hline & Medio & 1,33 & $0,71-2,53$ & 0,37 \\
\hline & Alto & 0,73 & $0,33-1,60$ & 0,43 \\
\hline \multirow{2}{*}{ Consumo de carnes rojas cocidas } & $\leq 250$ g/día & Referencia & & \\
\hline & $>250 \mathrm{~g} /$ día & 0,76 & $0,37-1,53$ & 0,44 \\
\hline \multirow{2}{*}{ Consumo de etanol } & $\leq 20 \mathrm{~g} / \mathrm{dí} a$ & Referencia & & \\
\hline & $>20 \mathrm{~g} / \mathrm{dí}$ & 1,16 & $0,67-2,00$ & 0,59 \\
\hline \multirow{2}{*}{ Nivel socioeconómico } & Medio-Alto & Referencia & & \\
\hline & Bajo & 1,74 & $1,04-2,90$ & 0,03 \\
\hline \multirow{2}{*}{ Nivel de actividad física } & Activo & Referencia & & \\
\hline & Sedentario & 1,18 & $0,71-1,96$ & 0,51 \\
\hline \multirow{3}{*}{ Valor energético total (VET) } & Tercil inferior & Referencia & & \\
\hline & Tercil medio & 1,10 & $0,52-2,29$ & 0,81 \\
\hline & Tercil superior & 3,07 & $1,27-7,43$ & 0,01 \\
\hline \multirow{2}{*}{ Índice de Masa Corporal (IMC) } & No sobrepeso $\left(<25 \mathrm{~kg} / \mathrm{m}^{2}\right)$ & Referencia & & \\
\hline & Sobrepeso $\left(\geq 25 \mathrm{~kg} / \mathrm{m}^{2}\right)$ & 0,91 & & 0,73 \\
\hline
\end{tabular}

física en cuanto al consumo de fibra alimentaria y la patología. La variable hábito de fumar no fue identificada como variable de confusión. Controlando los efectos de con- fusión identificados, en el análisis de riesgos alimentarios para la enfermedad, no se encontró asociación de la ingesta de fibra con la patología, y se observó un efecto promo- 
tor del nivel socioeconómico bajo y de la ingesta energética diaria.

El cáncer se ha convertido en un problema de salud pública en todo el mundo, ${ }^{9}$ y se prevé que las muertes por esta enfermedad sigan aumentando, ${ }^{2}$ por lo cual se considera importante conducir investigaciones que aborden el estudio de esta patología desde múltiples perspectivas, incluso respecto a aspectos metodológicos y en relación a factores alimentarios. Específicamente, en relación al CCR existe evidencia de que el consumo de carnes rojas, carnes procesadas y alcohol aumentan el riesgo de padecer la patología. También se ha observado una asociación inversa con el consumo de alimentos ricos en fibra, calcio, consumo de leche y ajo. ${ }^{1}$

Si bien la relación entre factores alimentarios y CCR es ampliamente reportada, se destaca que son escasos los trabajos que abordan simultáneamente el estudio del efecto confundente de variables como el NSE, actividad física y hábito de fumar, que pueden intervenir en la asociación observada. De hecho, la bibliografía reporta escasos antecedentes sobre la temática de la confusión en la estimación de riesgos, más aún en el campo de la epidemiología nutricional del cáncer. No obstante, se encontraron algunos estudios en los cuales se incluyen las variables de confusión del presente trabajo como variables de ajuste, pero sin profundizar sobre los aspectos metodológicos que justifican la selección de las mismas. Es relevante destacar la importancia de la identificación de factores confundentes en el estudio de la relación dieta-cáncer, para incluirlos en investigaciones posteriores en la población estudiada, mejorando las estimaciones de riesgo obtenidas. Así, existen investigaciones que consideraron la actividad física como variable de ajuste en el estudio de la asociación entre la ingesta alimentaria y el CCR. Como ejemplos puede citarse un estudio de cohorte sobre consumo de carne roja y el riesgo de cáncer colorrectal, realizado en Estados Unidos, ${ }^{38}$ y otro, de tipo caso-control, conducido en Grecia. ${ }^{39}$ También otros autores han analizado la ingesta de granos integrales (fuente de fibra dietética) ${ }^{40,41} \mathrm{y}$ de carnes rojas, ${ }^{42}$ ajustando sus análisis por nivel socioeconómico. Otros estudios han investigado la relación entre el consumo de alcohol y CCR, incluyendo como variable de ajuste el hábito de fumar. ${ }^{43,44}$

Específicamente en estudios observacionales de tipo caso-control, como el presente, se recomienda controlar la influencia de variables confundentes tanto en el diseño del estudio como en el análisis de los datos, para mejorar la validez de las estimaciones obtenidas. ${ }^{16}$ En este caso, luego de la identificación de factores confundentes (es decir, con comprobado efecto interviniente en la relación cáncer-dieta), se realizaron estimaciones de riesgo para CCR mediante modelos de regresión logística múltiple. La inclusión de las variables confundentes identificadas (nivel socioeconómico y actividad física) en el análisis de la relación de factores alimentarios específicos y el CCR permitiría obtener estimaciones de riesgo, que se acercan aún más a los verdaderos valores de efecto causal de esta asociación. ${ }^{45}$

Los resultados obtenidos en relación a la ingesta de fibra dietética indican que no existe asociación con el CCR. Diversos estudios como el Nurses Health Study y Cochrane, ${ }^{46}$, ${ }^{47}$ coinciden con estos hallazgos. Por otro lado, otras investigaciones ${ }^{12,47}$ encontraron asociación inversa entre esta variable y la aparición de la patología. También el European Prospective Investigation into Cancer and Nutrition (EPIC), estudio de ámbito mundial que incluyó casi 520 mil personas con un seguimiento durante seis años, relacionó inversamente la ingesta de fibra en dosis altas con la incidencia de cáncer de intestino grueso.

En cuanto a la ingesta de carnes rojas cocidas, numerosos estudios, ${ }^{8,38,46,48}$ demuestran una asociación positiva con la incidencia de CCR. La investigación realizada por Navarro y cols., ${ }^{48}$ en la ciudad de Córdoba (Argentina), sostiene que existe asociación positiva entre el consumo de carne roja asada con costra y la aparición de CCR. El consumo de este tipo de carnes se asocia con aumento en la dieta de aminas aromáticas heterocíclicas e hidrocarburos aromáticos policíclicos, considerados factores carcinógenos. ${ }^{8}$ Otro factor que se reconoce como fuertemente 
asociado al desarrollo de cáncer es el consumo de etanol. ${ }^{1} \mathrm{La}$ evidencia sugiere que los mecanismos por los cuales el alcohol actuaría como carcinógeno serían el incremento de la proliferación mucosa, la activación de sustancias procancerígenas en el intestino, cambios en la composición de la bilis y elevación de la concentración de nitrosaminas. ${ }^{1,11}$ En nuestro estudio se puede destacar que más del 30\% de los sujetos presentaron un consumo mayor a $250 \mathrm{~g} /$ día de carnes rojas cocidas, y que algo más de un tercio de la muestra presentó un consumo mayor a $20 \mathrm{~g} /$ día. A pesar de ello, la asociación con la enfermedad no pudo ser demostrada. Esto podría explicarse debido a la distribución homogénea observada entre casos y controles respecto a estas variables de consumo mencionadas.

Para concluir destacamos que se verificaron las hipótesis planteadas respecto al efecto confusor de la variable nivel socioeconómico en la relación entre la ingesta de carnes rojas cocidas, fibra y etanol y el CCR, y de la actividad física en cuanto al consumo de fibra alimentaria y la patología. Esto demandaría su consideración en futuros análisis de riesgo que involucren las mencionadas variables con el fin de obtener estimaciones precisas, que se aproximen a los verdaderos valores de efecto causal de esta asociación.

\section{CONCLUSIÓN}

Además de las variables alimentarias de exposición que se han reportado como asociadas a la ocurrencia de cáncer, existen factores confundentes que mezclan su efecto en la variable respuesta (ocurrencia de CCR). Desconocer la influencia de estos factores en el diseño metodológico puede producir sesgos en el análisis de los datos obtenidos, conduciendo a conclusiones erróneas. Se reconoce de suma importancia la identificación previa de las variables de confusión, con el fin de mejorar las estimaciones estadísticas en el estudio de la relación entre factores alimentarios y cáncer, y así llegar a realizar recomendaciones alimentarias certeras para promover hábitos alimentarios saludables en la población.

\section{REFERENCIAS}

1. World Cancer Research Fund. American Institute for Cancer Research. Food, nutrition, physical activity, and the prevention of cancer: a global perspective. Washington DC: American Institute for Cancer Research; 2007.

2. World Health Organization. Media Center. Cancer [en línea] WHO; c2014 [actualizado 17.02.2014; consultado 18.04.2012]. Disponible en: http://www.who.int/mediacentre/ factsheets/fs297/en/

3. Somoza MI, Torresani ME. Lineamientos para el cuidado nutricional. Bs. As.: EUDEBA; 2003, pp. 209-216.

4. Diaz MP, Corrente JE, Osella AR, Muñoz SE, Aballay LR. Modelling spatial distribution of cancer incidence in Cordoba, Argentina. Appl Cancer Res. 2010; 30(2): 245-252.

5. American Cáncer Society [en línea] 2014 [consultado 1.03.2011]. Disponible en: http://www.cancer.org/

6. International Agency for Research on Cancer. WHO. Globocan 2008: Colorectal Cancer Incidence, Mortality and Prevalence Worldwide in 2008 [en línea] [consultado 11.10.2012]. Disponible en: http://globocan. iarc.fr/Default.aspx

7. Díaz MP, Osella AR, Aballay LR et al. Cancer incidence pattern in Cordoba, Argentina. European J Cancer Prev. 2009; 18: 259-266.

8. Johnson IT, Lund EK. Institute of Food Research, Norwich Research Park, Colney, Norwich, UK. Review article: nutrition, obesity and colorectal cancer. Aliment Pharmacol Ther. 2007; 26(2): 161-81.

9. Verdú JM, Martínez JJ. Review article: Nutrition, obesity and colorectal cancer, vol. II. Publicación de Océano/ergon; 2002, pp. 1766-1789.

10. Rohrmann S, Hermann S, Lineisen J. Heterocyclic aromatic amine intake increases colorectal adenoma risk: findings from a prospective European cohort study. Am J Clin Nutr. 2009; 89(5): 1418-24.

11. Moskal A, Norat T, Ferrari P, Riboli E. Alcohol intake and colorectal cancer risk: A dose-response meta-analysis of published 
cohort studies. Int J Cancer. 2007; 120(3): 664-671.

12. Peters U, Sinha R, Chatterjee $N$ et al. Dietary fibre and colorectal adenoma in a colorectal cancer early detection programme. Lancet. 2003 May 3; 361(9368): 1491-5.

13. Kac G, Sichieri R, Petrucci Gigante D. Epidemiologia nutricional. Río de Janeiro: Fiocruz/Atheneu; 2007.

14. Willet W. Nutritional epidemiology. $2^{\mathrm{a}}$ ed. New York: Oxford University Press; 1998.

15. Szklo M, Nieto FJ. Epidemiology: beyond the basics. $2^{\mathrm{a}}$ ed. Burlington: Jones and Bartlett Published; 2007.

16. Lash TL, Fox MP, Fink AK. Applying quantitative bias analysis to epidemiologic studies. New York: Springer, Statistics for Biology and Health; 2009.

17. Rothman KJ, Greenland S. Modern Epidemiology. $2^{\mathrm{a}}$ ed. Philadelphia: LippincottRaven; 1998.

18. National Cancer Institut (U.S.). Physical Activity and Cancer [en línea]; 2009 [consultado 14.04.2011]. Disponible en: http:// www.cancer.gov/cancertopics/factsheet/ prevention/physicalactivity.

19. Moore HG. Colorectal cancer: what should patients and families be told to lower the risk of colorectal cancer? Surg Oncol Clin N Am. 2010; 19(4): 693-710.

20. de Vries E, Soerjomataram I, Lemmens VE, Coebergh JW, Barendregt JJ, Oenema A, et. al. Lifestyle changes and reduction of colon cancer incidence in Europe: A scenario study of physical activity promotion and weight reduction. Eur J Cancer. 2010; 46(14): 2605-16.

21. Slattery ML, Potter J, Caan B, Edwards S, Coates A, Ma KN, Berry TD. Energy balance and colon cancer-beyond physical activity. Cancer Res. 1997; 1; 57(1): 75-80.

22. American Cancer Society. Alcohol and Cancer [el línea] American Cancer Society; 2007 [consultado 7.04.2011]. Disponible en: http://www.cancer.org/acs/groups/content/@healthpromotions/documents/document/acsq-017622.pdf

23. Fedirko V, Tramacere I, Bagnardi V, Rota M, Scotti L, Islami F et al. Alcohol drinking and colorectal cancer risk: an overall and dose- response meta-analysis of published studies. Cancer Prev Res. 2011; 4(12): 2035-2043.

24. Navarro A, Muñoz S, Lantieri M, Díaz MP, Cristaldo P, Fabro S, et al. Meat cooking habits and risk of colorectal cancer in Córdoba, Argentina. Nutrition. 2004; 20(10): 873-877.

25. Matos E, Brandani A. Review on meat consumption and cancer in South America. $M u-$ tat Res. 2002; 506-507: 243-9.

26. Andreatta MM, Navarro A, Muñoz SE, Aballay L, Eynard AR. Dietary patterns and food groups are linked to the risk of urinary tract tumors in Argentina. Eur $J$ Cancer Prev. 2010; 19: 478-484.

27. Navarro A, Osella AR, Muñoz SE, Lantieri MJ, Fabro EA, Eynard AR. Fatty acids, fibres and colorectal cancer risk in Córdoba, Argentina. J Epidemiol Biostat. 1998; 4: 415.

28. Pou SA, Niclis C, Aballay LR, Tumas N, Román MD, Muñoz SE, Becaria Coquet J, Díaz MP. Cáncer y su asociación con patrones alimentarios en Córdoba. Nutr Hosp. 2014 (en prensa).

29. Pou SA, Díaz MP, Osella AR. Applying multilevel model to the relationship of dietary patterns and colorectal cancer: an ongoing case-control study in Córdoba, Argentina. Eur J Nutr. 2012; 51: 755-764.

30. Woodward M. Epidemiology: study design and data analysis. USA: Chapman \& Hall/ CRC. 1999.

31. Navarro A, Osella AR, Guerra V, et al. Reproducibility and validity of a food frequency questionnaire in assessing dietary intake and food habits in epidemiological cancer studies in Argentina.J Exp Clin Cancer Res. 2001. Sep; 20(3): 365-70.

32. Navarro A, Cristaldo PE, Díaz MP, Eynard AR. Atlas fotográfico para cuantificar el consumo de alimentos y nutrientes en estudios epidemiológicos en Córdoba, Argentina. Rev. Fac. Cienc. Méd. (Córdoba). 2007; 57: 67-74.

33. Peyrano M, Gigena J, Muñoz SE, Lantieri M, Eynard AR, Navarro A. A computer software system for the analysis of Dietary data in cancer epidemiological research. Presentado en el 17th International Cancer Congress. Bologna: Monduzzi Editore. pp. 381-384. 1998. 
34. International Physical Activity Cuestionnaire. Guidelines for data processing and análisis of the International Physical Activity Cuestionnaire (IPAQ). Short and long forms [en línea] 2005 [consultado 3.05.2011]. Disponible en: http://www.ipaq. ki.se/scoring.pdf

35. World Health Organization. Global Database on Body Mass Index. BMI Classification [en línea]. [consultado 7.01.2014.]. Disponible en: http://apps.who.int/bmi/ index.jsp?introPage=intro_3.html

36. British Medical Association. Handbook of Medical Ethics. London. 1980.

37. Mc Cullagh P. Quasi-Likelihood functions. Ann Stat. 1883; 11: 59-67.

38. Cross AJ, Leitzmann MF, Gail MH, Hollenbeck AR, Schatzkin A, Sinha R. A Prospective Study of Red and Processed Meat Intake in Relation to Cancer Risk. PLoS Med. 2007; (12): e325.

39. Karagianni V, Merikas E, Georgopoulos F. et al.Risk factors for colorectal polyps: findings from a Greek case-control study. Rev Med Chir Soc Med Nat Iasi 2010; 114(3): 662-70.

40. Kyro C, Skeie G, Dragsted LO, Christensen J, Overvad K, Hallmans G. Intake of whole grains in Scandinavia is associated with healthy lifestyle, socio-economic and dietary factors. Public Health Nutr. 2011; (10): 1787-95.

41. Kyro C, Olsen A, Skeie G, Dragsted LO. Intake and determinants of intake of whole grain foods in the Norway, Sweden and
Denmark. Nordic Health - Whole Grain Food [en línea]. [consultado 9.03.2012]. Disponible en: http://helgawholegrain.org/ resabstracts/kyro1.php.

42. Kim D, Masyn KE, Kawachi I, Laden F, Colditz GA. Neighborhood socioeconomic status and behavioral pathways to risks of colon and rectal cancer in women. Cancer. 2010; num. 1; 116(17): 4187-96.

43. Crockett SD, Long MD, Dellon ES, Martin CF, Galanko JA, Sandler RS. Inverse relationship between moderate alcohol intake and rectal cancer: analysis of the North Carolina Colon Cancer Study. Dis Colon Rectum. 2011; 54(7): 887-94.

44. Fu Z, Shrubsole MJ, Smalley WE et al. Lifestyle factors and their combined impact on the risk of colorectal polyps. Am J Epidemiol. 2012; 176(9): 766-76.

45. Weisberg HI. Bias and causation: Models and judgment for valid comparisons. New Jersey: John Wiley \& Sons, Inc.; 2012.

46. Escudero Álvarez E, González Sánchez P. La fibra dietética. Nutr Hosp. 2006; 21(2): 61-72.

47. Park Y, Hunter DJ, Spiegelman D, Bergkvist $\mathrm{L}$, Berrino $\mathrm{F}$, van den Brandt PA, et al. Dietary fiber intake and risk of colorectal cancer: a pooled analysis of prospective cohort studies. JAMA. 2005 Dec 14; 294(22): 2849-57.

48. Navarro A, Muñoz SE, Eynard AR. Diet, feeding habits and risk of colorectal cancer in Córdoba. Argentina. J Exp Clin Cancer Res. 1995; 14(3): 287-291. 